\title{
Highly Enantioselective Hiyama Cross-Coupling via Rh-Catalyzed Allylic Arylation of Racemic Allyl Chlorides
}

\author{
Jesús González, Philipp Schäfer, and Stephen P. Fletcher*10 \\ Chemistry Research Laboratory, University of Oxford, 12 Mansfield Road, Oxford OX1 3TA, United Kingdom
}

Supporting Information

ABSTRACT: Highly enantioselective Hiyama cross-coupling reactions have been achieved through rhodium(I)-catalyzed dynamic kinetic asymmetric transformations between aryl siloxanes and cyclic racemic allyl halides. This process affords valuable enantiomerically enriched arylsubstituted cyclic allyl products and is compatible with heterocyclic allyl chloride electrophiles.

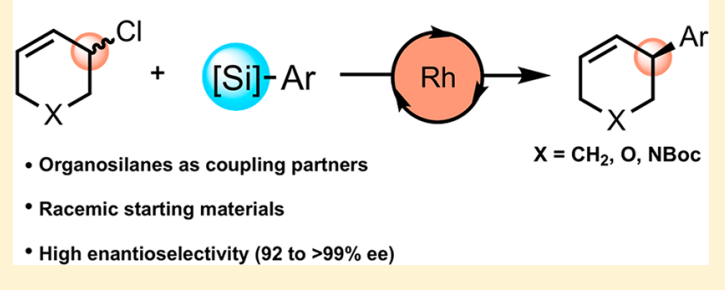

\section{INTRODUCTION}

Cross-coupling reactions occupy a privileged place among the tools to assemble carbon-based molecules, ${ }^{1}$ and SuzukiMiyaura, Negishi, Heck, and related reactions have been established as routine procedures. In general, the development of $\mathrm{C}-\mathrm{C}$ bond forming cross-coupling reactions has mainly focused on the construction of $\mathrm{Csp}^{2}-\mathrm{Csp}^{2}$ bonds, ${ }^{2}$ while the formation of $\mathrm{Csp}^{3}-\mathrm{Csp}^{2}$ bonds is more elusive. ${ }^{3,4}$

Hiyama cross-coupling procedures have been developed as the use of organosilicon coupling partners is attractive. ${ }^{5}$ Nontoxicity, stability, and ease of handling are features that promise to make organosilanes useful in an array of synthetic applications. Moreover, along with studies of their reactivity and applications, novel approaches for the preparation of organosilanes comprise an area of active research. ${ }^{6}$

In the context of asymmetric transformations, most processes involving organosilanes are Hosomi-Sakurai-type allylation reactions. ${ }^{7} \mathrm{Oi}$ and Inoue have reported $\mathrm{Rh}$-catalyzed asymmetric 1,4-addition of siloxanes to $\alpha, \beta$-unsaturated compounds (Scheme 1a). ${ }^{8}$ In cross-coupling processes, enantiospecific Hiyama couplings have been reported. ${ }^{9}$ However, to the best of our knowledge, only one example of enantioselective Hiyama-type coupling has been reported to date. In that work, Fu and co-workers developed a Ni-catalyzed coupling between $\alpha$-bromoesters and aryl- or vinylsiloxanes to prepare highly enantioenriched $\alpha$-functionalized esters (Scheme 1b). ${ }^{10}$

We became interested in the development of new dynamic kinetic asymmetric transformations (DYKATs), ${ }^{11}$ and have reported Rh-catalyzed Suzuki-Miyaura type procedures where boronic acids are coupled to racemic cyclic allyl halides. ${ }^{11 c, d}$ Here we extend catalytic asymmetric $\mathrm{Csp}^{2}-\mathrm{Csp}^{3}$ bond forming cross-coupling procedures to Hiyama-type process, employing arylsilanes as coupling partners (Scheme 1c). These reactions use racemic allyl halides as starting materials and appear to induce enantioselectivity through a DYKAT type mechanism. $^{12}$
Scheme 1. Relevant Enantioselective Transformations with Organosilanes $^{8,10}$

a) Rh-catalyzed asymmetric 1,4-addition of siloxanes (Oi, Inoue, 2003)

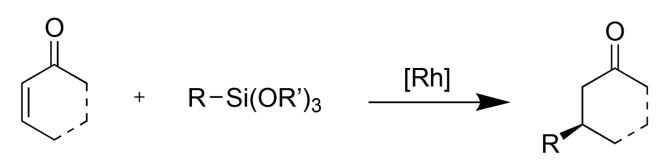

b) Ni-catalyzed asymmetric Hiyama cross-coupling (Fu, 2008)



c) Rh-catalyzed asymmetric Hiyama cross-coupling (This work)

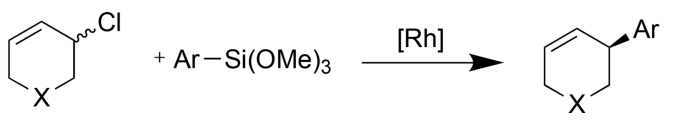

\section{RESULTS AND DISCUSSION}

We first evaluated racemic 3-chlorocyclohex-1-ene 1a in combination with silicon-based coupling partners that have previously been used in Hiyama couplings (Table 1). In these initial studies we used the cationic rhodium(I) complex $\left[\mathrm{Rh}(\mathrm{COD})(\mathrm{MeCN})_{2}\right]\left[\mathrm{BF}_{4}\right](5 \mathrm{~mol} \%)$ and $(S)$-BINAP (6 $\mathrm{mol} \%)$ as the ligand in THF at $60^{\circ} \mathrm{C}$. Under these conditions, the Hiyama-Denmark protocol ${ }^{13}$ involving a silanol and a bulky base did not give rise to desired product (entry 1 ). The use of trisiloxane was also unsuccessful (entries 2 and 3 ). Fortunately, we found that phenyl triethoxysilane in combina-

Special Issue: Asymmetric Synthesis Enabled by Organometallic Complexes

Received: March 22, 2019 
Table 1. Organosilane Screening for the $\mathrm{Rh}$-Catalyzed Allylic Arylation ${ }^{a}$

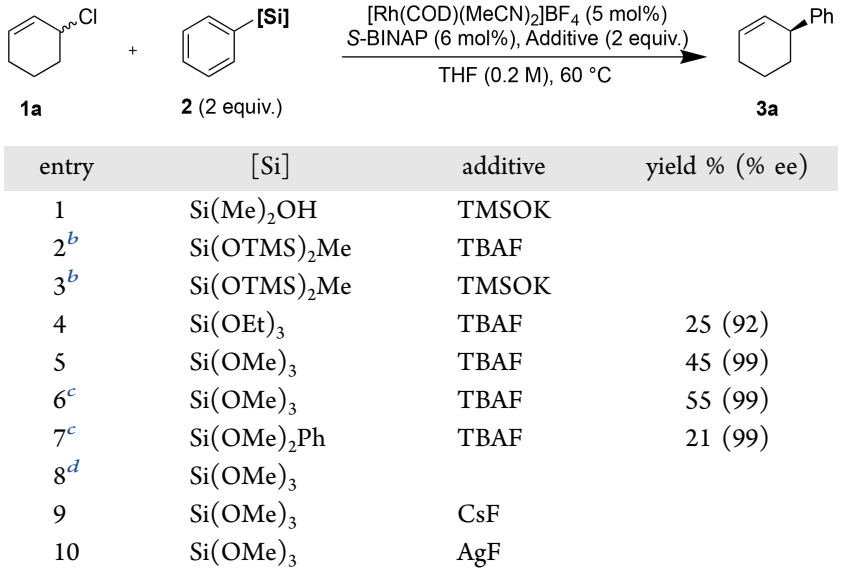

${ }^{a}$ Isolated yields after purification by chromatographic column. Enantioselectivity determined by HPLC. ${ }^{b}$ Using 1 equiv of organosilane and 3 equiv of additive. ${ }^{c}$ Reaction carried out at reflux. ${ }^{d}$ Reaction carried out in 1,4 -dioxane $/ \mathrm{H}_{2} \mathrm{O}$ at $90{ }^{\circ} \mathrm{C}$.

tion with TBAF afforded the desired product in low yield but good enantioselectivity (entry $4,25 \%$ yield, $92 \%$ ee). Both the yield and the enantioselectivity improved when phenyl trimethoxysilane was employed (entry 5), and carrying out the reaction at reflux gave higher yield (55\% yield, $99 \%$ ee, entry 6). Dimethoxydiphenylsilane showed the desired reactivity and excellent enantioselectivity but the yield was quite low ( $21 \%$ yield, $99 \%$ ee, entry 7 ). Interestingly, the Rhcatalyzed asymmetric 1,4-addition of organosilanes ${ }^{8 a}$ uses fluoride-free conditions, and when similar conditions were tried here we did not observe any desired product (entry 8). Finally, the use of alternative fluoride sources such as CsF or $\mathrm{AgF}$ did not give rise to $3 \mathrm{a}$ (entries 9 and 10).

We then proceeded to optimize further the conditions with the aim of improving the reaction yield (Table 2). As above, heating the reaction mixture to reflux in THF in the presence of $\left[\mathrm{Rh}(\mathrm{COD})(\mathrm{MeCN})_{2}\right]\left[\mathrm{BF}_{4}\right](5 \mathrm{~mol} \%),(S)$-BINAP (6 mol $\%$ ), and TBAF ( 2 equiv) gave $3 a$ in $55 \%$ yield with $99 \%$ ee (entry 1). Using SEGPHOS as ligand afforded similar results (entry 2), whereas the employment of other bisphosphine ligands led to diminished yields, although maintaining complete enantioselectivity (entries 3 and 4). When dienetype ligand L3 was tested, no trace of 3a was detected (entry 5). ${ }^{14}$ We were able to slightly improve the yield by increasing the metal/ligand ratio up to $1: 1.5$. Thus, $7.5 \mathrm{~mol} \%(S)$-BINAP gave $3 a$ in $65 \%$ yield (entry 6 ). Carrying out the reaction in 1,4-dioxane at $90{ }^{\circ} \mathrm{C}$ resulted in $11 \%$ yield (entry 7 ). Finally, other $\mathrm{Rh}(\mathrm{I})$ complexes were tested. However, none of them proved superior to the tetrafluoroborate salt (entries 8-11). ${ }^{15}$

Using the reaction conditions described in entry 6, Table 2 $\left\{\left[\mathrm{Rh}(\mathrm{COD})(\mathrm{MeCN})_{2}\right]\left[\mathrm{BF}_{4}\right](5 \mathrm{~mol} \%), S\right.$-BINAP $(7.5 \mathrm{~mol}$ $\%)$, TBAF ( 2 equiv), 2 equiv. siloxane in THF heated to reflux $\}$ we explored the scope of this transformation. Pleasingly, we found that the enantioselective $\mathrm{Rh}(\mathrm{I})$-catalyzed Hiyama cross-coupling with racemic allyl chlorides could be accomplished with several aryl siloxanes (Scheme 2). Different aromatic motifs such as naphthyl or biphenyl are also compatible, giving rise to the corresponding products $3 \mathbf{b}, \mathbf{c}$ in moderate yields and excellent enantioselectivity. The para- and meta-alkyl substituted arenes were tolerated, providing $\mathbf{3 d}-\mathbf{f}$
Table 2. Effect of Varying Reaction Conditions in the Enantioselective Cross-Coupling of $1 \mathrm{a}$ and $2 \mathrm{a}^{a}$

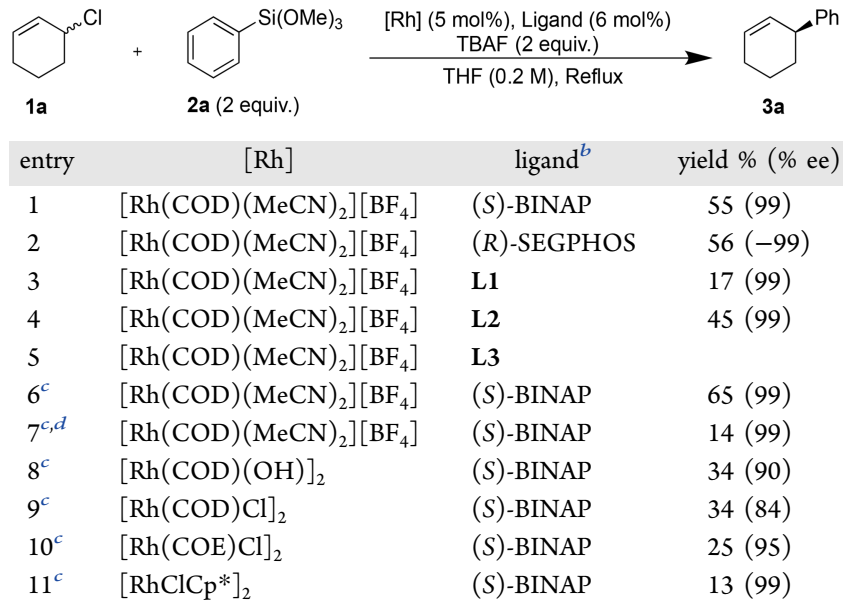

${ }^{a}$ Isolated yields after purification by chromatographic column. Enantioselectivity determined by HPLC. ${ }^{b}$
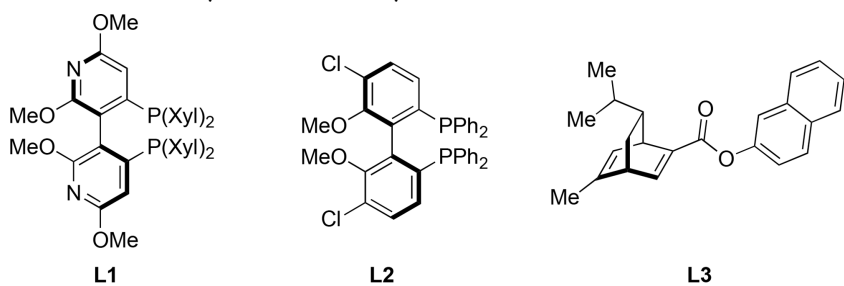

${ }^{c}$ Reaction carried out with $7.5 \mathrm{~mol} \%$ of ligand. ${ }^{d}$ Reaction carried out in 1,4-dioxane at $90{ }^{\circ} \mathrm{C}$.

with similar results in terms of both yield and enantioselectivity. In contrast, the use of an ortho-methyl substituted substrate only afforded traces of the desired product (not shown, $<5 \%$ yield). A para-methoxy group slightly diminished the yield, although maintaining great enantioselectivity (98\%) (3g). A similar effect was observed for the thioanisole derivative, leading to $34 \%$ yield and $95 \%$ ee ( $3 \mathrm{~h}$ ). However, with a methoxy group in the meta position, the desired product can be obtained in 51\% yield and $96 \%$ ee (3i). Furthermore, a 1,3-dioxole moiety can be used to prepare $3 \mathbf{j}$ with high enantioselectivity ( $99 \%$ ee). In addition, halogen-substituted aryl siloxanes were suitable coupling partners, affording the products $3 \mathbf{k}-\mathbf{n}$ with near complete enantioselectivity $(97 \rightarrow$ $99 \%$ ee).

Regarding heteroaryl siloxanes, commercially available triethoxy(thiophen-2-yl)silane was tested under these reaction conditions, but no product was detected. Additionally, some vinyl siloxanes were also tried, and only traces of product were observed in some cases.

Despite the promise of organosilicon coupling partners in synthesis, a limitation of this method is that there are currently few general robust procedures for the synthesis of aryltrimethoxysilanes, preventing the use of more elaborately functionalized silane coupling partners. However, we anticipate that if such aryl species were available then many of them would be tolerated in this reaction. We hope researchers in the field will develop methods to prepare such silanes.

Next, we examined if this protocol is applicable to heterocyclic allyl chloride coupling partners (Scheme 3). Using 3-chloro-3,6-dihydro-2H-pyran $\mathbf{1 b}$ with the conditions described above allowed us to prepare compound $3 \mathrm{~m}$ with 
Scheme 2. Rhodium-Catalyzed Enantioselective Synthesis of Tetrahydro Biphenyls $3 a-n^{a, b}$
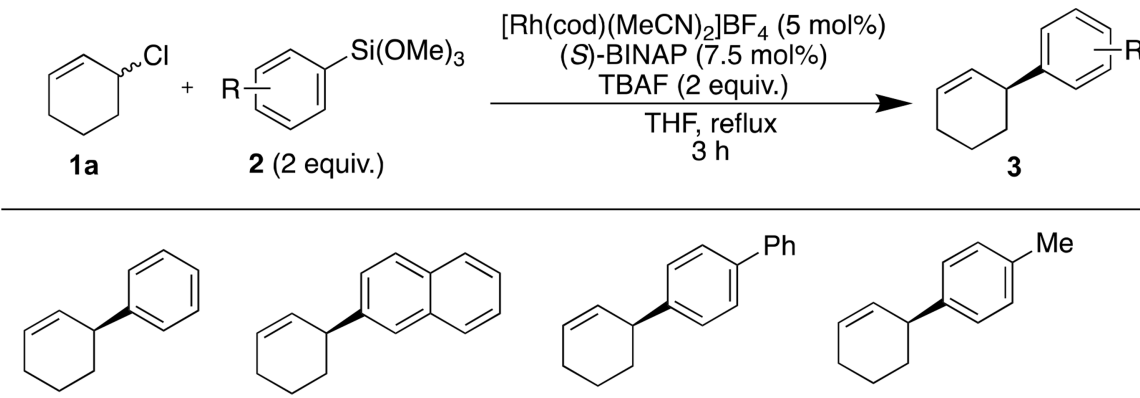

3a $(65 \%,>99 \%$ ee)

3b $(52 \%, 99 \%$ ee)

3c $(40 \%, 98 \%$ ee)

3d $(53 \%, 95 \%$ ee $)$<smiles>Cc1cccc(C2C=CCCC2)c1</smiles><smiles>Cc1cc(C)cc(C2C=CCCC2)c1</smiles><smiles>Cc1ccc(C2C=CCCC2)cc1</smiles><smiles>COc1cccc([C]2C=CCCC2)c1</smiles>

3e $(48 \%, 98 \%$ ee $)$

3f $(42 \%,>99 \%$ ee $)$

$\mathbf{3 g}(X=0,32 \%, 92 \% \text { ee })^{b}$
$\mathbf{3 h}(X=S, 34 \%, 95 \% \text { ee })^{b}$<smiles>C1=CC(c2ccc3c(c2)OCO3)CCC1</smiles>

3j $(48 \%, 99 \%$ ee $)$<smiles>[R]c1ccc(C2C=CCCC2)cc1</smiles>

3k $(R=F, 51 \%, 97 \%$ ee) 3I ( $\mathrm{R}=\mathrm{Br}, 34 \%, 99 \%$ ee $)$<smiles>Fc1cccc(C2C=CCCC2)c1</smiles>

$3 \mathrm{~m}\left(55 \%,>99 \%\right.$ ee) 3 n $(54 \%,>99 \% \text { ee })^{\mathrm{b}}$

${ }^{a}$ Reaction conditions: 1a $(0.4 \mathrm{mmol}), 2$ (2 equiv), TBAF ( 2 equiv), $\left[\mathrm{Rh}(\mathrm{cod})(\mathrm{MeCN})_{2}\right][\mathrm{BF} 4](5 \mathrm{~mol} \%),(S)$-BINAP $(7.5 \mathrm{~mol} \%)$, and THF $(0.2$ $\mathrm{M})$ at reflux. Yields correspond to isolated products. Enantioselectiviy was determined by HPLC or SFC. ${ }^{b}$ Contains minor impurities inseparable by flash column chromatography.

\section{Scheme 3. Rhodium-Catalyzed Enantioselective Hiyama Coupling with Heterocyclic Racemic Chlorides ${ }^{a}$}

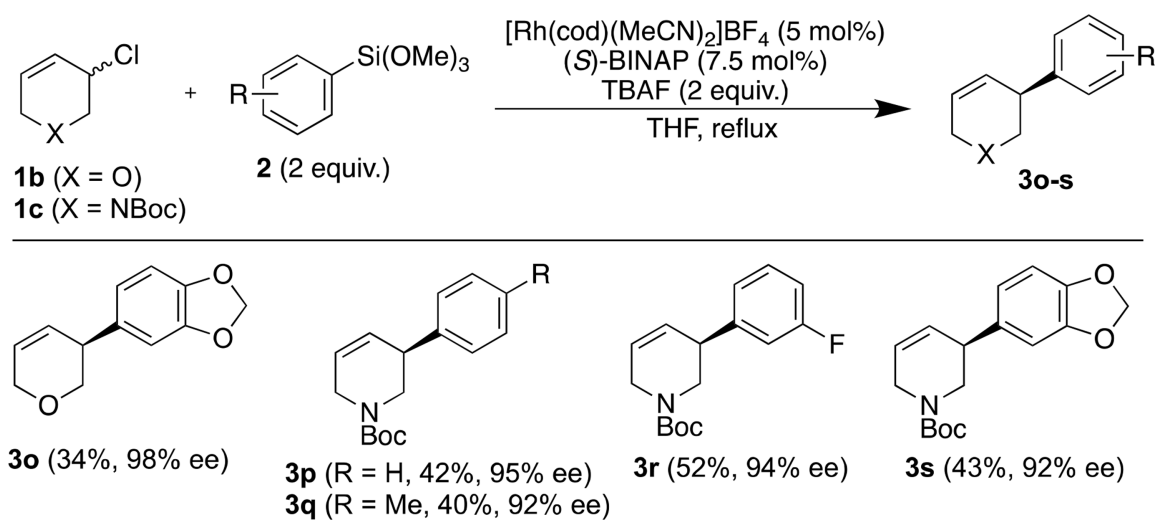

${ }^{a}$ Reactions conditions: 1 (0.4 mmol), 2 (2 equiv), TBAF (2 equiv), [ Rh(cod)(MeCN)2][BF4] (5 mol \%), (S)-BINAP (7.5 mol \%), THF (0.2 M) at reflux. Yields correspond to isolated products. Enantioselectiviy was determined by HPLC or SFC.

high enantioselectivity (98\%), albeit with only $34 \%$ yield. The synthesis of piperidine derivatives holds a significant importance within chemistry as it is present in numerous natural alkaloids, pharmaceuticals, and various synthetic substances with important properties. ${ }^{15}$ Therefore, we decided to apply this asymmetric Hiyama cross-coupling in the synthesis of enantioenriched dihydropiperidine derivatives using racemic $N$-Boc-protected allyl chloride $1 \mathrm{c}$ in combination with different arylsiloxanes. We were pleased to find that this process gave rise to compounds $3 \mathbf{n}-\mathbf{q}$ with high levels of enantiomeric excess.

During this study, variable amounts of the homocoupling and proto-demetalation products of the silane were detected.
Additionally, $\beta$-elimination on the allyl chloride could sometimes be observed. These competing processes probably account for the moderate yields of the coupling products.

Regarding the reaction mechanism, we tentatively hypothesize the catalytic cycle operates as outlined in Scheme 4. In contrast to our work with boronic acid nucleophiles, ${ }^{11 \mathrm{c}}$ the use of a cationic $\mathrm{Rh}$ source with a $\mathrm{BF}_{4}{ }^{-}$counterion gave the best results. We suggest that initial activation of silicon by fluoride sets the stage for $\mathrm{Si}$ to $\mathrm{Rh}$ transmetalation. Then, oxidative addition to the allyl chloride likely takes place to provide a $\mathrm{Rh}$ (III) species. This intermediate could equilibrate between diastereomeric Rh-allyl species through suprafacial 1,3-isomerization. ${ }^{16}$ If reductive elimination takes place preferentially in 
Scheme 4. Proposed Mechanism

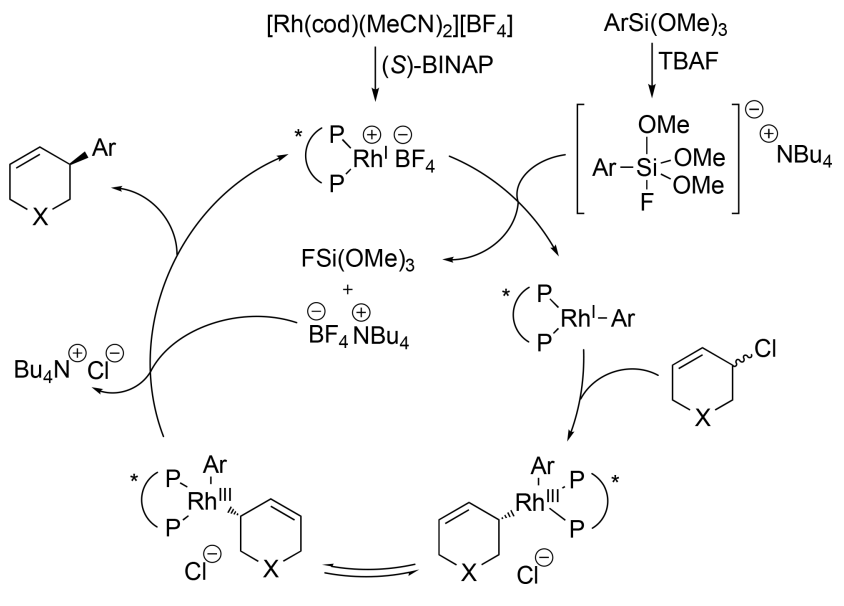

one of the isomers, then this would lead to the enantioenriched cross-coupling products 3 .

\section{CONCLUSION}

In summary, we have reported highly enantioselective Rhcatalyzed cross-couplings between arylsiloxanes and racemic cyclic allyl chlorides. This process represents a rare example of asymmetric Hiyama coupling, The method enables the preparation of valuable allyl arenes with uniformly high enantioselectivity (92 $\rightarrow 99 \%$ ee). Important heterocyclic scaffolds are compatible with this transformation, leading to highly enantioenriched dihydropyran and piperidine derivatives. At this stage, it is proposed that diastereoselective 1,3isomerization between two competing $\mathrm{Rh}-\sigma$-allyl species accounts for enantioselection.

\section{ASSOCIATED CONTENT}

\section{S Supporting Information}

The Supporting Information is available free of charge on the ACS Publications website at DOI: 10.1021/acs.organomet.9b00197.

Experimental procedures and characterization data (PDF)

\section{AUTHOR INFORMATION}

\section{Corresponding Author}

*E-mail: stephen.fletcher@chem.ox.ac.uk.

ORCID $\odot$

Stephen P. Fletcher: 0000-0001-7629-0997

Notes

The authors declare no competing financial interest.

\section{ACKNOWLEDGMENTS}

Financial support from the UK Engineering and Physical Sciences Research Council (EP/N022246/1) is gratefully acknowledged. J.G. thanks the “The European Union's Horizon 2020 research and innovation programme" for a Marie Skłodowska-Curie fellowship (GA 700108). P.S. thanks the People Programme (Marie Curie Actions) of the European Union's Seventh Framework Programme (FP7/2007-2013) under REA grant agreement 316955 for funding.

\section{REFERENCES}

(1) (a) De Meijere, A., Brässe, S., Oestreich, M., Eds. MetalCatalyzed Cross-Coupling Reactions and More; Wiley-VCH: Weinheim, 2014. (b) De Meijere, A., Diederich, F., Eds. Metal-Catalyzed CrossCoupling Reactions, 2nd ed.; Wiley-VCH: Weinheim, 2004. (c) Johansson Seechurn, C. C. C.; Kitching, M. O.; Colacot, T. J.; Snieckus, V. Palladium-catalyzed cross-coupling: a historical contextual perspective to the 2010 Nobel Prize. Angew. Chem., Int. Ed. 2012, 51, 5062-5085. (d) Nicolaou, K. C.; Bulger, P. G.; Sarlah, D. Palladium-catalyzed cross-coupling reactions in total synthesis. Angew. Chem., Int. Ed. 2005, 44, 4442-4489.

(2) (a) Cepanec, I., Ed. Synthesis of Biaryls; Elsevier: Oxford, 2004. (b) Hassan, J.; Sévignon, M.; Gozzi, C.; Schulz, E.; Lemaire, M. ArylAryl Bond Formation One Century after the Discovery of the Ullmann Reaction. Chem. Rev. 2002, 102, 1359-1470.

(3) For a review, see (a) Jana, R.; Pathak, T. P.; Sigman, M. S. Advances in Transition Metal (Pd, Ni, Fe)-Catalyzed Cross-Coupling Reactions Using Alkyl-organometallics as Reaction Partners. Chem. Rev. 2011, 111, 1417-1492. For a recent example, see (b) Guo, L.; Hsiao, C. C.; Yue, H.; Liu, X.; Rueping, M. Nickel-Catalyzed $\mathrm{C}_{\mathrm{sp2} 2}-\mathrm{C}_{\mathrm{sp} 3}$ Cross-Coupling via C-O Bond Activation. ACS Catal. 2016, 6, 44384442.

(4) For a review on enantioselective cross-coupling reactions, see Cherney, A. H.; Kadunce, N. T.; Reisman, S. E. Enantioselective and Enantiospecific Transition-Metal-Catalyzed Cross-Coupling Reactions of Organometallic Reagents To Construct C-C Bonds. Chem. Rev. 2015, 115, 9587-9652.

(5) (a) Komiyama, T.; Minami, Y.; Hiyama, T. Recent Advances in Transition-Metal-Catalyzed Synthetic Transformations of Organosilicon Reagents. ACS Catal. 2017, 7, 631-651. (b) Foubelo, F.; Nájera, C.; Yus, M. The Hiyama Cross-Coupling Reaction: New Discoveries. Chem. Rec. 2016, 16, 2521-2533. (c) Sore, H. F.; Galloway, W. R. J. D.; Spring, D. R. Palladium-catalysed crosscoupling of organosilicon reagents. Chem. Soc. Rev. 2012, 41, 18451865. (d) Nakao, Y.; Hiyama, T. Silicon-based cross-coupling reaction: an environmentally benign version. Chem. Soc. Rev. 2011, 40, 4893-4901.

(6) Selected examples: (a) Cheng, C.; Hartwig, J. F. IridiumCatalyzed Silylation of Aryl C-H Bonds. J. Am. Chem. Soc. 2015, 137, 592-595. (b) Toutov, A. A.; Liu, W.-B.; Betz, K. N.; Fedorov, A.; Stoltz, B. M.; Grubbs, R. H. Silylation of C-H bonds in aromatic heterocycles by an Earth-abundant metal catalyst. Nature 2015, 518, 80-84. (c) Cheng, C.; Hartwig, J. F. Rhodium-catalyzed intermolecular $\mathrm{C}-\mathrm{H}$ silylation of arenes with high steric regiocontrol. Science 2014, 343, 853-857.

(7) Denmark, S. E.; Fu, J. Catalytic Enantioselective Addition of Allylic Organometallic Reagents to Aldehydes and Ketones. Chem. Rev. 2003, 103, 2763-2794.

(8) (a) Oi, S.; Taira, A.; Honma, Y.; Inoue, Y. Asymmetric 1, 4Addition of Organosiloxanes to $\alpha, \beta$-Unsaturated Carbonyl Compounds Catalyzed by a Chiral Rhodium Complex. Org. Lett. 2003, 5, 97-99. For a recent review on Rh-catalyzed 1,4-additions, see (b) Heravi, M. M.; Dehghani, M.; Zadsirjan, V. Rh-catalyzed asymmetric 1, 4-addition reactions to $\alpha, \beta$-unsaturated carbonyl and related compounds: an update. Tetrahedron: Asymmetry 2016, 27, 513-588.

(9) (a) Denmark, S. E.; Werner, N. S. On the Stereochemical Course of Palladium-Catalyzed Cross-Coupling of Allylic Silanolate Salts with Aromatic Bromides. J. Am. Chem. Soc. 2010, 132, 36123620. (b) Hatanaka, Y.; Goda, K.; Hiyama, T. Regio- and stereoselective cross-coupling reaction of optically active allylsilanes: Stereocontrol of palladium-mediated $\mathrm{S}_{\mathrm{E}}{ }^{\prime}$ reactions. Tetrahedron Lett. 1994, 35, 1279-1282. (c) Hatanaka, Y.; Hiyama, T. Stereochemistry of the cross-coupling reaction of chiral alkylsilanes with aryl triflates: a novel approach to optically active compounds. J. Am. Chem. Soc. 1990, 112, 7793-7794.

(10) Dai, X.; Strotman, N. A.; Fu, G. C. Catalytic Asymmetric Hiyama Cross-Couplings of Racemic $\alpha$-Bromo Esters. J. Am. Chem. Soc. 2008, 130, 3302-3303. 
(11) (a) Schäfer, P.; Sidera, M.; Palacin, T.; Fletcher, S. P. Asymmetric cross-coupling of alkyl, alkenyl and (hetero)aryl nucleophiles with racemic allyl halides. Chem. Commun. 2017, 53, 12499-12511. (b) Rideau, E.; You, H.; Sidera, M.; Claridge, T. D. W.; Fletcher, S. P. Mechanistic Studies on a Cu-Catalyzed Asymmetric Allylic Alkylation with Cyclic Racemic Starting Materials. J. Am. Chem. Soc. 2017, 139, 5614-5617. (c) Schäfer, P.; Palacin, T.; Sidera, M.; Fletcher, S. P. Asymmetric Suzuki-Miyaura coupling of heterocycles via Rhodium-catalysed allylic arylation of racemates. Nat. Commun. 2017, 8, 15762-15769. (d) Sidera, M.; Fletcher, S. P. Rhodium-catalysed asymmetric allylic arylation of racemic halides with arylboronic acids. Nat. Chem. 2015, 7, 935-939. (e) Sidera, M.; Fletcher, S. P. Cu-catalyzed asymmetric addition of $\mathrm{sp}^{2}$-hybridized zirconium nucleophiles to racemic allyl bromides. Chem. Commun. 2015, 51, 5044-5047. (f) Rideau, E.; Fletcher, S. P. Copper-catalysed asymmetric allylic alkylation of alkylzirconocenes to racemic 3, 6dihydro-2H-pyrans. Beilstein J. Org. Chem. 2015, 11, 2435-2443. (g) You, H.; Rideau, E.; Sidera, M.; Fletcher, S. P. Non-stabilized nucleophiles in $\mathrm{Cu}$-catalysed dynamic kinetic asymmetric allylic alkylation. Nature 2015, 517, 351-355.

(12) A previous version of this manuscript has been deposited on a preprint server: Gonzalez, J.; Schafer, P.; Fletcher, S. P. Highly Enantioselective Hiyama Cross-Coupling via Rh-Catalyzed Allylic Arylation of Racemic Allyl Chlorides. 2019, chemrxiv.6960404.v1. chemrxiv.org e-Print archive. https://doi.org/10.26434/chemrxiv. 6960404.v1.

(13) Denmark, S. E.; Regens, C. S. Palladium-Catalyzed CrossCoupling Reactions of Organosilanols and Their Salts: Practical Alternatives to Boron- and Tin-Based Methods. Acc. Chem. Res. 2008, 41, 1486-1495.

(14) Biphenyl, which likely arises from homocoupling, was detected as the major product.

(15) (a) Vo, C. V. T.; Bode, J. W. Synthesis of Saturated NHeterocycles. J. Org. Chem. 2014, 79, 2809-2815. (b) Källström, S.; Leino, R. Synthesis of pharmaceutically active compounds containing a disubstituted piperidine framework. Bioorg. Med. Chem. 2008, 16, 601-635.

(16) (a) Wucher, B.; Moser, M.; Schumacher, S. A.; Rominger, F.; Kunz, D. First X-ray structure analyses of rhodium(III) $\eta^{1}$-allyl complexes and a mechanism for allylic isomerization reactions. Angew. Chem., Int. Ed. 2009, 48, 4417-4421. (b) Evans, P. A.; Nelson, J. D. Conservation of Absolute Configuration in the Acyclic RhodiumCatalyzed Allylic Alkylation Reaction: Evidence for an Enyl $(\sigma+\pi)$ Organorhodium Intermediate. J. Am. Chem. Soc. 1998, 120, 55815582 . 